\title{
MULTIPLIERS IN SOBOLEV SPACES AND EXACT CONVERGENCE RATE ESTIMATES FOR THE FINITE-DIFFERENCE SCHEMES
}

\author{
BOŠKO JOVANOVIĆ \\ University of Belgrade, Faculty of Sciences \\ Studentski trg 16, POB 550, 11000 Belgrade, Yugoslavia
}

\begin{abstract}
In this paper we present some recent results concerning convergence rate estimates for finite-difference schemes approximating boundary-value problems. Special attention is given to the problem of minimal smoothness of coefficients in partial differential equations necessary for obtaining the results.
\end{abstract}

1. Introduction. Recently, increased attention is given to approximation of generalized solutions of partial differential equations with finite-difference methods.

For a problem with the solution belonging to the Sobolev space $W_{p}^{s}(\Omega)$, the convergence estimate

$$
\|u-v\|_{W_{p}^{k}(\omega)} \leq C h^{s-k}\|u\|_{W_{p}^{s}(\Omega)}, \quad s>k,
$$

is said to be compatible with the smoothness of the solution [12]. Here $u \in W_{p}^{s}(\Omega)$ denotes the solution of the original boundary-value problem, $v$ denotes the solution of the corresponding finite-difference scheme, $h$ is the discretization parameter, $W_{p}^{k}(\omega)$ denotes the discrete Sobolev space, and $C$ is a positive generic constant, independent of $h$ and $u$.

Estimates of this type have been obtained for a broad class of elliptic problems (see $[6,10,11,13,16])$. Analogous results have also been obtained for parabolic and hyperbolic problems (see $[5,7,8,9])$. As a rule, the Bramble-Hilbert lemma $[2,4]$ is used in their proofs.

1991 Mathematics Subject Classification: 46E35, 65N15.

Key words and phrases: Sobolev spaces, multipliers, boundary-value problems, finite differences.

This work was supported by SF of Serbia, grant number 0401F.

The paper is in final form and no version of it will be published elsewhere. 
For equations with variable coefficients, the natural problem arises of establishing the minimal smoothness properties of coefficients for obtaining the same type (1) of estimate. Such coefficients belong to classes of multipliers in Sobolev spaces.

2. Multipliers in Sobolev spaces. Let $\Omega$ be a domain in $\mathbb{R}^{n}$. By $D(\Omega)=$ $\dot{C}^{\infty}(\Omega)$ we denote the space of infinitely smooth functions with compact support in $\Omega$, and by $D^{\prime}(\Omega)$ the space of distributions. Moreover, $x=\left(x_{1}, x_{2}, \ldots, x_{n}\right)$ denote vectors from $\mathbb{R}^{n}$, and $\alpha=\left(\alpha_{1}, \alpha_{2}, \ldots, \alpha_{n}\right)$ are multi-indices. Let $|\alpha|=$ $\alpha_{1}+\alpha_{2}+\ldots+\alpha_{n}$. Partial derivatives are denoted by

$$
D_{i} u=\partial u / \partial x_{i} \quad \text { and } \quad D^{\alpha} u=D_{1}^{\alpha_{1}} D_{2}^{\alpha_{2}} \ldots D_{n}^{\alpha_{n}} u
$$

Suppose $V$ and $W$ are two function spaces contained in $D^{\prime}(\Omega)$. A function $a$ defined on $\Omega$ is called a pointwise multiplier, or simply a multiplier, from $V$ to $W$ if, for every $v$ in $V$, the product $a \cdot v$ belongs to $W$. The set of all multipliers from $V$ to $W$ is denoted by $M(V \rightarrow W)$. In particular, when $V=W$ we put $M(V)=M(V \rightarrow V)$.

In this section, we shall be concerned with multipliers in Sobolev spaces which belong to $M\left(W_{p}^{t}(\Omega) \rightarrow W_{p}^{s}(\Omega)\right), 1<p<\infty$. Naturally, we assume that $t \geq s$.

To begin, we consider multipliers in pairs of Sobolev spaces on $\mathbb{R}^{n}$. Motivated by the definition of multiplication of a distribution with a smooth function, for $a \in M\left(W_{p}^{t}\left(\mathbb{R}^{n}\right) \rightarrow W_{p}^{s}\left(\mathbb{R}^{n}\right)\right)$ and $u \in W_{p^{\prime}}^{-s}\left(\mathbb{R}^{n}\right), 1 / p+1 / p^{\prime}=1$, we define the product $a \cdot u \in W_{p^{\prime}}^{-t}\left(\mathbb{R}^{n}\right)$ by

$$
\langle a \cdot u, \varphi\rangle_{W_{p^{\prime}}^{-t} \times W_{p}^{t}}=\langle u, a \cdot \varphi\rangle_{W_{p^{\prime}}^{-s} \times W_{p}^{s}}, \quad \forall \varphi \in W_{p}^{t}\left(\mathbb{R}^{n}\right) .
$$

This definition implies that $M\left(W_{p^{\prime}}^{-s}\left(\mathbb{R}^{n}\right) \rightarrow W_{p^{\prime}}^{-t}\left(\mathbb{R}^{n}\right)\right)=M\left(W_{p}^{t}\left(\mathbb{R}^{n}\right) \rightarrow W_{p}^{s}\left(\mathbb{R}^{n}\right)\right)$, and therefore it suffices to explore the properties of the sets $M\left(W_{p}^{t}\left(\mathbb{R}^{n}\right) \rightarrow\right.$ $\left.W_{p}^{s}\left(\mathbb{R}^{n}\right)\right)$ and $M\left(W_{p}^{t}\left(\mathbb{R}^{n}\right) \rightarrow W_{p}^{-s}\left(\mathbb{R}^{n}\right)\right)$ for $t \geq s \geq 0$

We recall a collection of fundamental results on multipliers in Sobolev spaces (see [14]).

LEMma 1. If $a \in M\left(W_{p}^{t}\left(\mathbb{R}^{n}\right) \rightarrow W_{p}^{s}\left(\mathbb{R}^{n}\right)\right), t \geq s \geq 0$, then:

$$
\begin{aligned}
a & \in M\left(W_{p}^{t-s}\left(\mathbb{R}^{n}\right) \rightarrow L_{p}\left(\mathbb{R}^{n}\right)\right), & & \\
a & \in M\left(W_{p}^{t-\sigma}\left(\mathbb{R}^{n}\right) \rightarrow W_{p}^{s-\sigma}\left(\mathbb{R}^{n}\right)\right), & & 0<\sigma<s, \\
D^{\alpha} a & \in M\left(W_{p}^{t}\left(\mathbb{R}^{n}\right) \rightarrow W_{p}^{s-|\alpha|}\left(\mathbb{R}^{n}\right)\right), & & |\alpha| \leq s, \\
D^{\alpha} a & \in M\left(W_{p}^{t-s+|\alpha|}\left(R^{n}\right) \rightarrow L_{p}\left(\mathbb{R}^{n}\right)\right), & & |\alpha| \leq s .
\end{aligned}
$$


Lemma 2. For $t \geq s \geq 0, M\left(W_{p}^{t}\left(\mathbb{R}^{n}\right) \rightarrow W_{p}^{s}\left(\mathbb{R}^{n}\right)\right) \subseteq W_{p \text {, unif }}^{s}$, where

$W_{p, \text { unif }}^{s}=\left\{f \mid \sup _{z \in \mathbb{R}^{n}}\|\eta(x-z) \cdot f(x)\|_{W_{p}^{s}}<\infty, \forall \eta \in D\left(\mathbb{R}^{n}\right), \eta \equiv 1\right.$ on $\left.B_{1}\right\}$,

and $B_{1}$ is the unit ball with center 0 . If tp $>n$, then $M\left(W_{p}^{t}\left(\mathbb{R}^{n}\right) \rightarrow W_{p}^{s}\left(\mathbb{R}^{n}\right)\right)=$ $W_{p, \text { unif }}^{s}$.

LEMMA 3. For $s \geq 0, M\left(W_{p}^{s}\left(\mathbb{R}^{n}\right)\right) \subseteq L_{\infty}\left(\mathbb{R}^{n}\right)$.

Lemma 4. Suppose $1<p<\infty$, and let $s$ and $t$ be nonnegative integers such that $t \geq s$. If

$$
a=\sum_{|\alpha| \leq \mathbf{t}} D^{\alpha} a_{\alpha}
$$

and $a_{\alpha} \in M\left(W_{p}^{t}\left(\mathbb{R}^{n}\right) \rightarrow W_{p}^{t-s}\left(\mathbb{R}^{n}\right)\right) \cap M\left(W_{p^{\prime}}^{s}\left(\mathbb{R}^{n}\right) \rightarrow L_{p^{\prime}}\left(\mathbb{R}^{n}\right)\right), 1 / p+1 / p^{\prime}=1$, then $a \in M\left(W_{p}^{t}\left(\mathbb{R}^{n}\right) \rightarrow W_{p}^{-s}\left(\mathbb{R}^{n}\right)\right)$.

LEMMA 5. Let $p>1, t>s>0$, and suppose that either $q \in[n / t, \infty]$ and tp $<n$, or $q \in(p, \infty)$ and $t p=n$. If

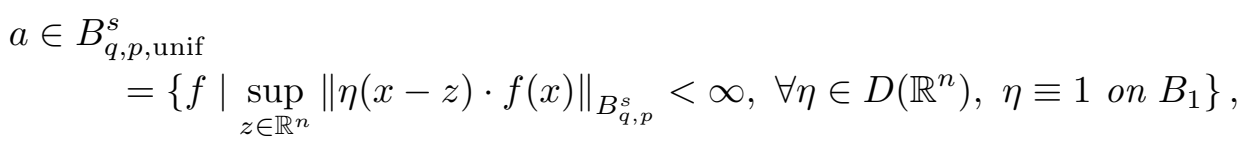

where $B_{q, p}^{s}$ is the Besov space, then $a \in M\left(W_{p}^{t}\left(\mathbb{R}^{n}\right) \rightarrow W_{p}^{s}\left(\mathbb{R}^{n}\right)\right)$. The result is also true for $t=s$, provided $a \in B_{q, p \text {,unif }}^{s} \cap L_{\infty}\left(\mathbb{R}^{n}\right)$.

LEMMA 6. If $a_{\alpha} \in M\left(W_{p}^{s-|\alpha|}\left(\mathbb{R}^{n}\right) \rightarrow W_{p}^{s-k}\left(\mathbb{R}^{n}\right)\right), s \geq k$, for every multi-index $\alpha$ then the differential operator

$$
L u=\sum_{|\alpha| \leq k} a_{\alpha}(x) D^{\alpha} u, \quad x \in \mathbb{R}^{n},
$$

defines a continuous mapping from $W_{p}^{s}\left(\mathbb{R}^{n}\right)$ to $W_{p}^{s-k}\left(\mathbb{R}^{n}\right)$.

The analogous result holds true for $s<0$. If $p=2$ then the result holds true for every $s$. Under certain conditions we have the converse result:

LemMa 7. Let the operator (2) define a continuous mapping from $W_{p}^{s}\left(\mathbb{R}^{n}\right)$ to $W_{p}^{s-k}\left(\mathbb{R}^{n}\right)$, and $p(s-k)>n, p>1$. Then $a_{\alpha} \in M\left(W_{p}^{s-|\alpha|}\left(\mathbb{R}^{n}\right) \rightarrow W_{p}^{s-k}\left(\mathbb{R}^{n}\right)\right)$, for every multi-index $\alpha$.

All of these results can be transfered to Sobolev spaces in an open subset of $\mathbb{R}^{n}$. More precisely, if $\Omega$ is an open set in $\mathbb{R}^{n}$ with a Lipschitz continuous boundary and $a \in M\left(W_{p}^{t}(\Omega) \rightarrow W_{p}^{s}(\Omega)\right)$, then $a$ can be extended to a function $\widetilde{a}$, defined on the whole of $\mathbb{R}^{n}$, such that $\widetilde{a} \in M\left(W_{p}^{t}\left(\mathbb{R}^{n}\right) \rightarrow W_{p}^{s}\left(\mathbb{R}^{n}\right)\right)$. The converse is also true: the restriction to $\Omega$ of a multiplier $a \in M\left(W_{p}^{t}\left(\mathbb{R}^{n}\right) \rightarrow W_{p}^{s}\left(\mathbb{R}^{n}\right)\right)$ is an element of $M\left(W_{p}^{t}(\Omega) \rightarrow W_{p}^{s}(\Omega)\right)$.

For bounded domains, $W_{p \text {,unif }}^{s}$ and $B_{q, p \text {,unif }}^{s}$ are replaced by standard Sobolev and Besov spaces, respectively. Employing Lemmas 2, 3, 5, imbedding theorems 
for Besov spaces $[1,17]$ and the representation of distributions from negative Sobolev spaces [18], we obtain the following results:

LEMMA 8. Suppose that $\Omega$ is a bounded open subset of $\mathbb{R}^{n}$ with a Lipschitz continuous boundary, $s>0$ and $p>1$. If $a \in W_{q}^{t}(\Omega)$ where

$$
\begin{aligned}
& q=p, t=s \quad \text { when } s p>n, \quad \text { and } \\
& q \geq n / s, t=s+\varepsilon, \varepsilon>0 \quad \text { when } s p \leq n,
\end{aligned}
$$

then $a \in M\left(W_{p}^{s}(\Omega)\right)$.

LEMMA 9. Let $\Omega$ be a bounded open set in $\mathbb{R}^{n}$ with a Lipschitz continuous boundary, $s>0$ and $p>1$. If $a \in L_{q}(\Omega)$ where

$$
\begin{array}{ll}
q=p & \text { when } s p>n, \\
q>p & \text { when } s p=n, \quad \text { and } \\
q \geq n / s & \text { when } s p<n,
\end{array}
$$

then $a \in M\left(W_{p}^{s}(\Omega) \rightarrow L_{p}(\Omega)\right)$.

LEMMA 10. Let $\Omega$ be a bounded open set in $\mathbb{R}^{n}$ with a Lipschitz continuous boundary and

$$
a(x)=a_{0}(x)+\sum_{i=1}^{n} D_{i} a_{i}(x) .
$$

If $a_{0} \in M\left(W_{2}^{t}(\Omega) \rightarrow L_{2}(\Omega)\right)$, and $a_{i} \in M\left(W_{2}^{t}(\Omega) \rightarrow W_{2}^{1-s}(\Omega)\right) \cap M\left(W_{2}^{t-1}(\Omega) \rightarrow\right.$ $\left.L_{2}(\Omega)\right), i=1,2, \ldots, n$, where $0<s \leq 1 \leq t<2, s \neq 1 / 2$, then $a \in M\left(W_{2}^{t}(\Omega) \rightarrow\right.$ $\left.W_{2}^{-s}(\Omega)\right)$.

3. Boundary-value problem and its approximation. As a model problem let us consider the first boundary-value problem for a second-order linear elliptic equation with variable coefficients, in the square $\Omega=(0,1)^{2}$ :

$$
-\sum_{i, j=1}^{2} D_{i}\left(a_{i j} D_{j} u\right)+a u=f \quad \text { in } \Omega, \quad u=0 \quad \text { on } \Gamma=\partial \Omega .
$$

We assume that the generalized solution of the problem (3) belongs to the Sobolev space $W_{2}^{s}(\Omega), 1<s \leq 3$, with the right-hand side $f(x)$ belonging to $W_{2}^{s-2}(\Omega)$. Consequently, the coefficients $a_{i j}(x)$ and $a(x)$ belong to the following classes of multipliers: $a_{i j} \in M\left(W_{2}^{s-1}(\Omega)\right), a \in M\left(W_{2}^{s}(\Omega) \rightarrow W_{2}^{s-2}(\Omega)\right)$. According to Lemmas 8-10 sufficient conditions are the following:

$$
\begin{aligned}
& a_{i j} \in W_{2}^{|s-1|}(\Omega), \quad a \in W_{2}^{|s-1|-1}(\Omega), \quad \text { for }|s-1|>1, \\
& a_{i j} \in W_{p}^{|s-1|+\delta}(\Omega), \quad a=a_{0}+\sum_{i=1}^{2} D_{i} a_{i}, \\
& a_{0} \in L_{2+\varepsilon}(\Omega), \quad a_{i} \in W_{p}^{|s-1|+\delta}(\Omega),
\end{aligned}
$$


where $\varepsilon>0$,

$$
\begin{aligned}
& \delta>0, \quad p>2 /|s-1| \quad \text { for } 0<|s-1| \leq 1, \quad \text { and } \\
& \delta=0, \quad p=\infty \quad \text { for } s=1 .
\end{aligned}
$$

The following estimates do not depend on $\delta$ in any way, so we can put $\delta=0$.

We also assume that the following conditions hold:

$$
\begin{gathered}
a_{i j}=a_{j i}, \quad \sum_{i, j=1}^{2} a_{i j} y_{i} y_{j} \leq c_{0} \sum_{i=1}^{2} y_{i}^{2}, \quad x \in \Omega, \quad c_{0}=\text { const }>0, \\
a(x) \geq 0 \quad \text { in the sense of distributions, i.e. } \\
\langle a \cdot \varphi, \varphi\rangle_{D^{\prime} \times D} \geq 0, \quad \forall \varphi \in D(\Omega) .
\end{gathered}
$$

Let $\bar{\omega}$ be the uniform mesh in $\bar{\Omega}$ with step $h, \omega=\bar{\omega} \cap \Omega, \gamma=\bar{\omega} \cap \Gamma, \gamma_{i k}=$ $\left\{x \in \gamma \mid x_{i}=k, 0<x_{3-i}<1\right\}, k=0,1$, and $\omega_{i}=\omega \cup \gamma_{i 0}$. We define finite differences as usual:

$$
v_{x_{i}}=\left(v^{+i}-v\right) / h, \quad v_{\bar{x}_{i}}=\left(v-v^{-i}\right) / h,
$$

where $v^{ \pm i}(x)=v\left(x \pm h r_{i}\right)$, and $r_{i}$ is the unit vector on the $x_{i}$ axis.

We also define the Steklov smoothing operators:

$$
T_{i}^{+} f(x)=\int_{0}^{1} f\left(x+h t r_{i}\right) d t=T_{i}^{-} f\left(x+h r_{i}\right)=T_{i} f\left(x+0.5 h r_{i}\right) .
$$

These operators commute and transform derivatives to differences:

$$
T_{i}^{+} D_{i} u=u_{x_{i}}, \quad T_{i}^{-} D_{i} u=u_{\bar{x}_{i}} .
$$

We approximate the problem (3) with the following finite-difference scheme:

$$
L_{h} v=T_{1}^{2} T_{2}^{2} f \quad \text { in } \omega, \quad v=0 \quad \text { on } \gamma
$$

where $L_{h} v=-0.5 \sum_{i, j=1}^{2}\left[\left(a_{i j} v_{\bar{x}_{j}}\right)_{x_{i}}+\left(a_{i j} v_{x_{j}}\right)_{\bar{x}_{i}}\right]+\left(T_{1}^{2} T_{2}^{2} a\right) v$ and $T_{i}^{2}=T_{i}^{+} T_{i}^{-}$. The difference scheme (4) is a standard symmetric difference scheme (see [15]) with the right-hand side and coefficient $a(x)$ averaged. For $1<s \leq 3$ these coefficients may not be continuous, so the difference scheme with non-averaged data is not well defined.

4. Convergence of the finite-difference scheme. Let $u$ denote the solution to the boundary value problem (3) and $v$ the solution to the difference scheme (4). The error $z=u-v$ satisfies the conditions

$$
L_{h} z=\sum_{i, j=1}^{2} \eta_{i j, \bar{x}_{i}}+\zeta \quad \text { in } \omega, \quad z=0 \quad \text { on } \gamma
$$

where $\eta_{i j}=T_{i}^{+} T_{3-i}^{2}\left(a_{i j} D_{j} u\right)-0.5\left(a_{i j} u_{x_{j}}+a_{i j}^{+i} u_{\bar{x}_{j}}^{+i}\right)$ and $\zeta=\left(T_{1}^{2} T_{2}^{2} a\right) u-T_{1}^{2} T_{2}^{2}(a u)$. 
For $\theta \subseteq \bar{\omega}$ let $(\cdot, \cdot)_{\theta}=(\cdot, \cdot)_{L_{2}(\theta)}$ and $\|\cdot\|_{\theta}=\|\cdot\|_{L_{2}(\theta)}$ denote the discrete inner product and the discrete $L_{2}$-norm on $\theta$. We also define the discrete $W_{2}^{1}$-norm on $\omega$ :

$$
\|v\|_{W_{2}^{1}(\omega)}^{2}=\|v\|_{\omega}^{2}+\left\|v_{x_{1}}\right\|_{\omega_{1}}^{2}+\left\|v_{x_{2}}\right\|_{\omega_{2}}^{2} .
$$

Using the energy method [15] it is easy to prove the next lemma.

LEMma 11. The finite-difference scheme (5) is stable in the sense of the a priori estimate

$$
\|z\|_{W_{2}^{1}(\omega)} \leq C\left(\sum_{i, j=1}^{2}\left\|\eta_{i j}\right\|_{\omega_{i}}+\|\zeta\|_{\omega}\right) .
$$

The problem of deriving the convergence rate estimate for the finite-difference scheme (4) is now reduced to estimating the right-hand side terms in (6). Estimates which follow are based on the following bilinear version of the BrambleHilbert lemma [3, 10]:

LEMMA 12. Let $E$ be a bounded open set in $\mathbb{R}^{n}$ with a Lipschitz continuous boundary and let $\eta(u, v)$ be a bounded bilinear functional on $W_{p}^{s}(E) \times W_{q}^{t}(E)$, $1 \leq p, q \leq \infty, s, t>0$, such that

$$
\eta(u, v)=0
$$

if either $u$ is a polynomial of degree $<s$ and $v \in W_{q}^{t}(E)$, or $v$ is a polynomial of degree $<t$ and $u \in W_{p}^{s}(E)$. Then there exists a positive constant $C=C(E, p, s, q, t)$ such that

$$
|\eta(u, v)| \leq|u|_{W_{p}^{s}(E)}|v|_{W_{q}^{t}(E)}, \quad \forall(u, v) \in W_{p}^{s}(E) \times W_{q}^{t}(E),
$$

with the seminorms of the corresponding spaces at the right-hand side.

First, we decompose $\eta_{i j}$ in the following way:

$$
\begin{gathered}
\eta_{i j}=\eta_{i j 1}+\eta_{i j 2}+\eta_{i j 3}+\eta_{i j 4}, \quad \text { where } \\
\eta_{i j 1}=T_{i}^{+} T_{3-i}^{2}\left(a_{i j} D_{j} u\right)-\left(T_{i}^{+} T_{3-i}^{2} a_{i j}\right) \cdot\left(T_{i}^{+} T_{3-i}^{2} D_{j} u\right), \\
\eta_{i j 2}=\left[T_{i}^{+} T_{3-i}^{2} a_{i j}-0.5\left(a_{i j}+a_{i j}^{+i}\right)\right] \cdot\left(T_{i}^{+} T_{3-i}^{2} D_{j} u\right), \\
\eta_{i j 3}=0.5\left(a_{i j}+a_{i j}^{+i}\right) \cdot\left[T_{i}^{+} T_{3-i}^{2} D_{j} u-0.5\left(u_{x_{j}}+u_{\bar{x}_{j}}^{+i}\right)\right], \quad \text { and } \\
\eta_{i j 4}=-0.25\left(a_{i j}-a_{i j}^{+i}\right) \cdot\left(u_{x_{j}}-u_{\bar{x}_{j}}^{+i}\right) .
\end{gathered}
$$

For $1<s \leq 2$ we set $\zeta=\zeta_{0}+\zeta_{1}+\zeta_{2}$, where

$$
\begin{aligned}
\zeta_{0} & =\left(T_{1}^{2} T_{2}^{2} a_{0}\right) u-T_{1}^{2} T_{2}^{2}\left(a_{0} u\right), \quad \text { and } \\
\zeta_{i} & =\left(T_{1}^{2} T_{2}^{2} D_{i} a_{i}\right) u-T_{1}^{2} T_{2}^{2}\left(D_{i} a_{i} \cdot u\right), \quad i=1,2 .
\end{aligned}
$$

For $2<s \leq 3$ we set $\zeta=\zeta_{3}+\zeta_{4}$, where

$$
\begin{aligned}
& \zeta_{3}=\left(T_{1}^{2} T_{2}^{2} a\right) \cdot\left(u-T_{1}^{2} T_{2}^{2} u\right), \quad \text { and } \\
& \zeta_{4}=\left(T_{1}^{2} T_{2}^{2} a\right) \cdot\left(T_{1}^{2} T_{2}^{2} u\right)-T_{1}^{2} T_{2}^{2}(a \cdot u) .
\end{aligned}
$$


Let us introduce the elementary rectangles $e=e(x)=\left\{y|| y_{j}-x_{j} \mid \leq h, j=\right.$ $1,2\}$ and $e_{i}=e_{i}(x)=\left\{y\left|x_{i} \leq y_{i} \leq x_{i}+h,\right| y_{3-i}-x_{3-i} \mid \leq h\right\}, i=1,2$.

The value $\eta_{i j 1}$ at the node $x \in \omega_{i}$ is a bounded bilinear functional on $W_{q}^{\lambda}\left(e_{i}\right) \times$ $W_{2 q /(q-2)}^{\mu}\left(e_{i}\right)$ where $\lambda \geq 0, \mu \geq 1$ and $q>2$. Moreover, $\eta_{i j 1}=0$ if either $a_{i j}$ is a constant or $u$ is a first-degree polynomial. Using Lemma 12 and a procedure proposed in [11], developed in [10], we obtain

$$
\left|\eta_{i j 1}\right| \leq C(h)\left|a_{i j}\right|_{W_{q}^{\lambda}\left(e_{i}\right)}|u|_{W_{2 q /(q-2)}^{\mu}\left(e_{i}\right)}, \quad 0 \leq \lambda \leq 1,1 \leq \mu \leq 2,
$$

where $C(h)=C h^{\lambda+\mu-2}$. Summation with the use of the Hölder inequality yields

$$
\left\|\eta_{i j 1}\right\|_{\omega_{i}} \leq C h^{\lambda+\mu-1}\left|a_{i j}\right|_{W_{q}^{\lambda}(\Omega)}|u|_{W_{2 q /(q-2)}^{\mu}(\Omega)}, \quad 0 \leq \lambda \leq 1,1 \leq \mu \leq 2 .
$$

Set $\lambda=s-1, \mu=1$ and $q=p$. By the imbedding theorem [17], $W_{2}^{s} \subseteq W_{2 p /(p-2)}^{1}$ for $1<s \leq 2$. Therefore, from (7) we obtain

$$
\left\|\eta_{i j 1}\right\|_{\omega_{i}} \leq C h^{s-1}\left\|a_{i j}\right\|_{W_{p}^{s-1}(\Omega)}\|u\|_{W_{2}^{s}(\Omega)}, \quad 1<s \leq 2 .
$$

Similar estimates hold for $\eta_{i j 2}, \eta_{i j 4}, \zeta_{1}$ and $\zeta_{2}$.

Let now $q>2$ be a constant. The following imbeddings hold: $W_{2}^{\lambda+\mu-1} \subseteq W_{q}^{\lambda}$ for $\mu>2-2 / q$, and $W_{2}^{\lambda+\mu} \subseteq W_{2 q /(q-2)}^{\mu}$ for $\lambda>2 / q$. Setting $\lambda+\mu=s$ we obtain from (7),

$$
\left\|\eta_{i j 1}\right\|_{\omega_{i}} \leq C h^{s-1}\left\|a_{i j}\right\|_{W_{2}^{s-1}(\Omega)}\|u\|_{W_{2}^{s}(\Omega)}, \quad 2<s \leq 3 .
$$

In the same manner we can estimate $\eta_{i j 4}$.

For $s>2, \eta_{i j 2}(x)$ is a bounded bilinear functional on $W_{2}^{s-1}\left(e_{i}\right) \times W_{\infty}^{1}\left(e_{i}\right)$ which vanishes if either $a_{i j}$ is a first-degree polynomial or $u$ is a constant. Using the same lemma and the imbedding $W_{2}^{s} \subseteq W_{\infty}^{1}$ we obtain for $\eta_{i j 2}$ an estimate of the form (9).

Similarly, $\eta_{i j 3}(x)$ is a bounded bilinear functional on $L_{\infty}\left(e_{i}\right) \times W_{2}^{s}\left(e_{i}\right), s>1$, which vanishes if $u$ is a second-degree polynomial. In the same way, using the imbeddings $W_{p}^{s-1} \subseteq L_{\infty}$ (for $1<s \leq 2$ ) and $W_{2}^{s-1} \subseteq L_{\infty}$ (for $s>2$ ) we again obtain estimates of the forms $(8)$ and $(9)$.

Let $2<q<2 /(3-s)$. For $2<s \leq 3, \zeta_{3}(x)$ is a bounded bilinear functional on $L_{q}(e) \times W_{2 q /(q-2)}^{s-1}(e)$. Moreover, $\zeta_{3}=0$ if $u$ is a first-degree polynomial. Using the Bramble-Hilbert lemma and the imbeddings $W_{2}^{s-2} \subseteq L_{q}$ and $W_{2}^{s} \subseteq W_{2 q /(q-2)}^{s-1}$ we obtain the estimate

$$
\left\|\zeta_{3}\right\|_{\omega} \leq C h^{s-1}\|a\|_{W_{2}^{s-2}(\Omega)}\|u\|_{W_{2}^{s}(\Omega)}, \quad 2<s \leq 3 .
$$

For $2<s \leq 3, \zeta_{4}(x)$ is a bounded bilinear functional on $W_{2}^{s-2}(e) \times W_{\infty}^{1}(e)$. Using the same methodology and the imbedding $W_{2}^{s} \subseteq W_{\infty}^{1}$, we obtain for $\zeta_{4}$ an estimate of the form (10).

Finally, let $2<q<\min \{2+\varepsilon, 2 /(2-s)\}$. For $1<s \leq 2, \zeta_{0}(x)$ is a bounded bilinear functional on $L_{q}(e) \times W_{2 q /(q-2)}^{s-1}(e)$ which vanishes if $u$ is a constant. Using 
the imbeddings $L_{2+\varepsilon} \subseteq L_{q}$ and $W_{2}^{s} \subseteq W_{2 q /(q-2)}^{s-1}$, we obtain the estimate

$$
\left\|\zeta_{0}\right\|_{\omega} \leq C h^{s-1}\left\|a_{0}\right\|_{L_{2+\varepsilon}(\Omega)}\|u\|_{W_{2}^{s}(\Omega)}, \quad 1<s \leq 2
$$

Combining (6) with (8)-(11) we obtain the final result:

THEOREM. The finite-difference scheme (4) converges and the following estimates hold:

$$
\begin{array}{r}
\|u-v\|_{W_{2}^{1}(\omega)} \leq C h^{s-1}\left(\max _{i, j}\left\|a_{i j}\right\|_{W_{2}^{s-1}(\Omega)}+\|a\|_{W_{2}^{s-2}(\Omega)}\right)\|u\|_{W_{2}^{s}(\Omega)}, \\
\text { for } 2<s \leq 3,
\end{array}
$$

and

$$
\begin{aligned}
\|u-v\|_{W_{2}^{1}(\omega)} \leq & C h^{s-1}\left(\max _{i, j}\left\|a_{i j}\right\|_{W_{p}^{s-1}(\Omega)}+\max _{i}\left\|a_{i}\right\|_{W_{p}^{s-1}(\Omega)}\right. \\
& \left.+\left\|a_{0}\right\|_{L_{2+\varepsilon}(\Omega)}\right)\|u\|_{W_{2}^{s}(\Omega)}, \quad \text { for } 1<s \leq 2 .
\end{aligned}
$$

The obtained convergence-rate estimates (12) and (13) are compatible with the smoothness of data. An analogous estimate in $L_{2}$-norm is obtained in [6]. Non-stationary problems were considered in $[7,8]$.

\section{References}

[1] R. A. Adams, Sobolev Spaces, Academic Press, New York 1975.

[2] J. H. Bramble and S. R. Hilbert, Bounds for a class of linear functionals with application to Hermite interpolation, Numer. Math. 16 (1971), 362-369.

[3] P. G. Ciarlet, The Finite Element Method for Elliptic Problems, North-Holland, Amsterdam 1978.

[4] T. Dupont and R. Scott, Polynomial approximation of functions in Sobolev spaces, Math. Comp. 34 (1980), 441-463.

[5] B. S. Jovanović, On the convergence of finite-difference schemes for parabolic equations with variable coefficients, Numer. Math. 54 (1989), 395-404.

[6] - Optimal error estimates for finite-difference schemes with variable coefficients, Z. Angew. Math. Mech. 70 (1990), 640-642.

[7] - Convergence of finite-difference schemes for parabolic equations with variable coefficients, ibid. 71 (1991), 647-650.

[8] - Convergence of finite-difference schemes for hyperbolic equations with variable coeffcients, ibid. 72 (1992), to appear.

[9] B. S. Jovanović, L. D. Ivanović and E. E. Süli, Convergence of a finite-difference scheme for second-order hyperbolic equations with variable coefficients, IMA J. Numer. Anal. 7 (1987), 39-45.

[10] - , - - C, Convergence of finite-difference schemes for elliptic equations with variable coefficients, ibid., 301-305.

[11] R. D. Lazarov, On the question of convergence of finite-difference schemes for generalized solutions of the Poisson equation, Differentsial'nye Uravneniya 17 (1981), 1285-1294 (in Russian).

[12] R. D. Lazarov, V. L. Makarov and A. A. Samarskiǔ, Application of exact difference schemes for construction and investigation of difference schemes for generalized solutions, Mat. Sb. 117 (1982), 469-480 (in Russian). 
[13] R. D. Lazarov, V. L. Makarov and W. Weinelt, On the convergence of difference schemes for the approximation of solutions $u \in W_{2}^{m}(m>0.5)$ of elliptic equations with mixed derivatives, Numer. Math. 44 (1984), 223-232.

[14] V. G. Maz'ya and T. O. Shaposhnikova, Theory of Multipliers in Spaces of Differentiable Functions, Monographs Stud. Math. 23, Pitman, Boston 1985.

[15] A. A. Samarskiŭ, Theory of Difference Schemes, Nauka, Moscow 1983 (in Russian).

[16] E. Süli, B. Jovanović and L. Ivanović, Finite difference approximations of generalized solutions, Math. Comp. 45 (1985), 319-327.

[17] H. Triebel, Interpolation Theory, Function Spaces, Differential Operators, Deutscher Verlag der Wissenschaften, Berlin 1978.

[18] J. Wloka, Partial Differential Equations, Cambridge Univ. Press, 1987. 